\title{
Search for critical points by measuring spatial correlation lengths via multiplicity density fluctuations
}

\section{Kensuke Homma* and PHENIX collaboration}

Hiroshima University, 1-3-1 Kagamiyama, Higashi-hiroshima, Hiroshima, Japan

E-mail: homma@hepl.hiroshima-u.ac.jp

\begin{abstract}
The nature of phase boundaries in the QCD phase diagram has not been satisfactorily explored by experiments. Based on the Ginzburg-Landau free energy with a spatially inhomogeneous term as a function of a scalar order parameter, it is possible to determine spatial correlation lengths from measured two point correlation functions in general, where a spatially dependent multiplicity fluctuation from the mean value is taken as the order parameter. The divergence of the correlation lengths can be a robust signature of a critical system as well as the disappearance of $<q \bar{q}>$ condensations. Simultaneous observations of such observables can probe the nature of the phase boundary conclusively. In this letter, a present result from the PHENIX experiment will be reported. We will focus whether a critical behavior of the phase transition exists or not by searching for increases of pseudo-rapidity correlation lengths as a function of the number of participant nucleons with dominantly low $p_{t}$ charged particles where instant fluctuations such as high $p_{t}$ jets are expected to be negligible. The result shows a non monotonic increase in the correlation lengths which can be a symptom of the critical behavior.
\end{abstract}

The 3rd edition of the International Workshop - The Critical Point and Onset of Deconfinement July 3-7 2006

Galileo Galilei Institute, Florence, Italy

\footnotetext{
${ }^{*}$ Speaker.
} 


\section{Introduction}

Many observations at RHIC indicate the formation of the strongly interacting dense medium with partonic natures[1]. Nevertheless, information on the phase boundaries have not been quantified. Although lattice QCD calculations as well as model calculations predict the existence of a tricritical end-point, none of them reaches a quantitative agreement each other on the location of the end-point[2]. Hence it is crucial to pin down the end-point in the QCD phase diagram and investigate the transition order by experiments. Before pinning down the end-point as an ultimate goal, it is important to establish ways to determine critical systems in general. As one of such observables, increases of spatial correlation lengths as a function of system energy density can be a robust signature to determine critical systems whatever the transition order is.

In the following sections the relation between the phase transition and the density fluctuations is briefly reviewed and the experimentally accessible observables are advocated. Based on the observables, Au+Au collision events taken by the PHENIX detector[3] at $\sqrt{s_{N N}}=200 \mathrm{GeV}$ have been analyzed and the present results are summarized by focusing on whether critical behaviors of the phase transition exist or not as a function of the number of participants $N_{p}$ which reflects the system energy density[4].

\section{Density fluctuation and phase transition}

In order to relate the density fluctuations with the phase transition in the simplest form, Ginzburg-Landau(GL)[5] theory with the Ornstein-Zernike picture[6] for a scalar order parameter is briefly reviewed. The first attempt to apply the free energy discussion to nucleus-nucleus collisions can be found in [7]. GL describes the relation between a free energy density $f$ and an order parameter $\phi$ as a function of system temperature $T$. By adding a spatially inhomogeneous term $(\nabla \phi)^{2}$ and an external field $h$, the general form is described as follows;

$$
\begin{array}{r}
f(T, \phi, h)=f_{0}(T)+\frac{1}{2} A(T)(\nabla \phi)^{2}+ \\
\frac{1}{2} a(T) \phi^{2}+\frac{1}{4} b \phi^{4}+\cdots-h \phi
\end{array}
$$

where terms with odd powers are neglected due to the symmetry of the order parameter and the sign of $b$ is used to classify the transition orders; $b<0$ for first order, $b>0$ for second order and $b=0$ for the critical point. Since the order parameter should vanish above critical temperature $T$, it is natural for the coefficient $a(T)$ to be expressed as $a(T)=a_{0}\left(T-T_{c}\right)$, while $b$ is usually assumed to be constant in the vicinity of $T_{c}$.

In the following analysis, the order parameter corresponds to the multiplicity density fluctuation from the mean density as a function of one dimensional rapidity point $y$, which is defined as

$$
\phi(y)=\rho(y)-\langle\rho\rangle
$$

where a pair of brackets is an operator to take the average. With the Fourier expansion of the density fluctuation $\phi(y)=\sum_{k} \phi_{k} e^{i k y}$ where $k$ is wave number, one can express the deviation of the 
free energy density $\Delta F / Y$ due to spatial fluctuations from the equilibrium value as

$$
\Delta F / Y=\frac{1}{Y} \int\left(f-f_{0}\right) d y=\frac{1}{2} \sum_{k}\left|\phi_{k}\right|^{2}\left(a(T)+A(T) k^{2}\right)
$$

where $Y$ is the total rapidity range corresponding to one dimensional volume and up to the second order terms are taken into account as an approximation in the vicinity of the critical point in Eq.(2.1). Given the free energy deviation, one can obtain the statistical weight $w$ for fluctuation $\phi(y)$ to occur in a given temperature $T$

$$
w(\phi(y))=N e^{-\Delta F / T} .
$$

Therefore the statistical average of the square of the density fluctuation with the wave number $k$ is described as

$$
\begin{array}{r}
\left\langle\left|\phi_{k}\right|^{2}\right\rangle=\int_{-\infty}^{+\infty}\left|\phi_{k}\right|^{2} w\left(\sum_{k} \phi_{k} e^{i k y}\right) d \phi_{k} \\
=\frac{N T}{Y} \frac{1}{a(T)+A(T) k^{2}} .
\end{array}
$$

Experimentally observable two point density correlation function can be related to the statistical average of the square of the density fluctuation. With a density $\rho\left(y_{i}\right)$ for a given sub volume $d y_{i}$, the general two point density correlation $G_{2}$ is expressed as

$$
G_{2}\left(y_{1}, y_{2}\right)=\left\langle\left(\rho\left(y_{1}\right)-\langle\rho\rangle\right)\left(\rho\left(y_{2}\right)-\langle\rho\rangle\right)\right\rangle
$$

where the case that 1 coincides with 2 is excluded to simplify the following discussion. Multiplying $e^{-i k y} \equiv e^{-i k\left(y_{2}-y_{1}\right)}$ to the both sides of Eq.(2.6) and integrating over sub volume $d y_{1}$ and $d y_{2}$ gives

$$
Y \int G_{2}(y) e^{-i k y} d y=\left\langle\left|\int(\rho(y)-\langle\rho\rangle) e^{-i k y} d y\right|^{2}\right\rangle=\left\langle\left|\phi_{k}\right|^{2}\right\rangle .
$$

From Eq.(2.5) and (2.7), $G_{2}$ can be obtained by the inverse Fourier transformation of $\left\langle\left|\phi_{k}\right|^{2}\right\rangle$. Therefore in the one dimensional case $G_{2}$ is described as

$$
G_{2}(y)=\frac{N T}{2 Y^{2} A(T)} \xi(T) e^{-|y| / \xi(T)},
$$

where a correlation length $\xi(T)$ is introduced, which is defined as

$$
\xi(T)^{2}=\frac{A(T)}{a_{0}\left(T-T_{c}\right)} .
$$

In general, a singular behavior of $\xi(T)$ as a function of $T$ indicates the critical point of the phase transition.

The wave number dependent susceptibility can also be defined from Eq.(2.1) and (2.3) as follows

$$
\begin{gathered}
\chi_{k}=-\left(\frac{\partial^{2} f}{\partial h^{2}}\right)_{T}=\left(\frac{\partial h}{\partial \phi_{k}}\right)^{-1}=\left(\frac{\partial^{2}(\Delta F / Y)}{\partial \phi_{k}^{2}}\right)^{-1} \\
=\frac{1}{a_{0}(T-T c)\left(1+k^{2} \xi(T)^{2}\right)}
\end{gathered}
$$


In the case of the static limit of $k=0$, the susceptibility can be expressed as

$$
\chi_{k=0}=\frac{1}{a_{0}(T-T c)}=\frac{2 Y^{2}}{N T} \xi(T) G_{2}(0) .
$$

From Eq.(2.9) and (2.11), simultaneous observations of singularities or discontinuous behaviors in both $\xi(T)$ and $\chi_{k=0} T \propto \xi(T) G_{2}(0)$ at the same temperature indicate that the phase transition is consistent with the second order transition by assuming continuous variations of $T$.

\section{Experimental observables}

In the following analysis the density fluctuation is discussed via the charged particle multiplicity distributions as a function of the pseudo-rapidity interval of $\delta \eta$ for each collision centrality. Let us introduce one and two particle inclusive multiplicity densities $\rho$ and $\rho_{2}$ based on the inclusive differential cross section with respect to the total inelastic cross section $q_{n e l}$ as follows[8]

$$
\begin{array}{r}
\frac{1}{\sigma_{\text {inel }}} d \sigma=\rho_{1}(\eta) d \eta \\
\frac{1}{\sigma_{\text {inel }}} d^{2} \sigma=\rho_{2}\left(\eta_{1}, \eta_{2}\right) d \eta_{1} d \eta_{2} .
\end{array}
$$

With these densities, a two particle density correlation function is defined as

$$
C_{2}\left(\eta_{1}, \eta_{2}\right)=\rho_{2}\left(\eta_{1}, \eta_{2}\right)-\rho_{1}\left(\eta_{1}\right) \rho_{1}\left(\eta_{2}\right) .
$$

The mathematical connection between second order normalized factorial moment $F_{2}$ and the two particle correlation function is expressed as[9]

$$
\begin{aligned}
F_{2}(\delta \eta)= & \frac{\langle n(n-1)\rangle}{\langle n\rangle^{2}}=\frac{\iint^{\delta \eta} \rho_{2}\left(\eta_{1}, \eta_{2}\right) d \eta_{1} d \eta_{2}}{\left\{\int^{\delta \eta} \rho_{1}(\eta) d \eta\right\}^{2}} \\
& =\frac{1}{(\delta \eta)^{2}} \iint^{\delta \eta} \frac{C_{2}\left(\eta_{1}, \eta_{2}\right)}{{\overline{\rho_{1}}}^{2}} d \eta_{1} d \eta_{2}+1
\end{aligned}
$$

where $n$ is the number of produced particles, $\delta \eta$ is the rapidity interval which defines the measurable range of $\left|\eta_{1}-\eta_{2}\right|, \overline{\rho_{1}}$ is the average number density per unit length within $\delta \eta$ which is defined as

$$
\overline{\rho_{1}}=\frac{1}{\delta \eta} \int^{\delta \eta} \rho_{1}(\eta) d \eta
$$

The two particle correlation function $C_{2}$ can be parametrized based on the one dimensional function form obtained in Eq.(2.8). However, one has to bear in mind that the damping behavior in Eq.(2.8) is originated only from the spatial inhomogeneity of the system in a fixed temperature. In many experimental conditions, the initial system temperature can not be specified as a point. For instance, corresponding temperature is indirectly discussed by relating it with the collision centrality. The centrality bin has a finite size and it causes fluctuations originating from the finite temperature bin size. In principle this kind of fluctuations must be independent of the spatial fluctuations. In addition, although a self correlation at the zero distance between the two sub 
volumes in Eq.(2.6) was excluded, the self correlation can not be excluded in the integrated two particle correlation function contained in Eq.(3.3), since there is no explicit distinction between two particles in the following analysis procedure. Therefore the general function form for the normalized two particle correlation function in the one dimensional analysis can be parameterized as follows which explicitly contains a constant term $\beta$;

$$
\frac{C_{2}\left(\eta_{1}, \eta_{2}\right)}{{\overline{\rho_{1}}}^{2}}=\alpha e^{-\delta \eta / \xi}+\beta
$$

where $\overline{\rho_{1}}$ is proportional to the mean multiplicity in each centrality.

Instead of $F_{2}$ itself, we will use an indirect parameter $k$ of the Negative Binomial Distribution(NBD) in the following analysis which is defined as

$$
P_{k, \mu}(n)=\frac{\Gamma(n+k)}{\Gamma(n-1) \Gamma(k)}\left(\frac{\mu / k}{1+\mu / k}\right) \frac{1}{1+\mu / k},
$$

where $\mu$ corresponds to the mean value and $k^{-1}$ reflects deviations from the completely random case i.e. the Poisson distribution which corresponds to $k=\infty$. Intuitively $k^{-1}$ is a measure how strongly particles are correlated. The mathematical relation between $k$ and $F_{2}$ is expressed as[10]

$$
k^{-1}=F_{2}-1 .
$$

The reason why we adopt NBD rather than $F_{2}$ is that NBD can provide an approximate probability distribution which enables us to estimate how inefficient or dead areas of the detector system bias the $k$ parameter and to obtain the true value of $k$ based on the estimation, while factorial moment itself does not provide any specific models on the distribution function which resulted the observed factorial moment.

As the result, the relation between the NBD $k$ parameter and the pseudo-rapidity interval $\delta \eta$ for the parametrization given in Eq.(3.5) is expressed as

$$
k^{-1}(\delta \eta)=F_{2}-1=\frac{2 \alpha \xi^{2}\left(\delta \eta / \xi-1+e^{-\delta \eta / \xi}\right)}{\delta \eta^{2}}+\beta .
$$

Once $\alpha, \xi$ and $\mu$ are obtained from the NBD fits, one can measure the product of the static susceptibility and the corresponding temperature based on Eq.(2.11) and (3.5);

$$
\chi_{k=0} T \propto{\overline{\rho_{1}}}^{2} \xi \alpha \equiv\left(\frac{\mu}{\mu_{\max }}\right)^{2} \xi \alpha
$$

where $\mu_{\max }$ is the mean multiplicity in the most central collision event sample in the following analysis. Experimentally it is enough to see how the $\chi_{k=0} T$ behaves as a function of a quantity which reflects $T$.

\section{Data analysis}

In the data analysis $258 \mathrm{k}$ events taken by a minimum bias trigger during Run2 were used. The trigger requires coincident hits in Beam-Beam Counters(BBC) and Zero-Degree Calorimeters. 


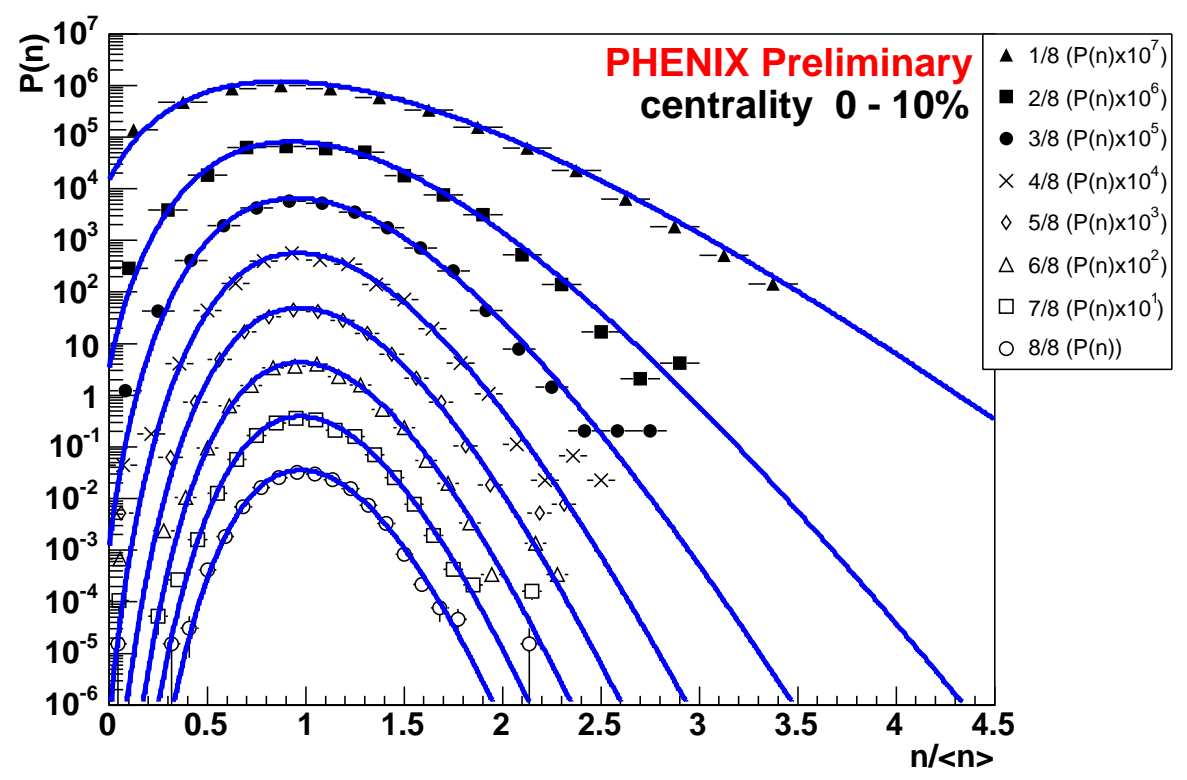

Figure 1: Multiplicity distributions in each $\delta \eta$ indicated inside the figure measured in $0-10 \%$ centrality bin in $\mathrm{Au}+\mathrm{Au}$ collisions at $\sqrt{s_{N N}}=200 \mathrm{GeV}$. The horizontal axis is normalized by the mean multiplicities. The vertical axis is scaled by the factors indicated inside the figure.

The charged particles were reconstructed by a drift chamber and two multi-wire chambers with pad readouts without a magnetic field in order to statistically enhance the low $p$ charged particles. It is essential to focus on the low $p_{t}$ charged pions for the discussion of the phase transition. The charged particles were measured by the east arm of the PHENIX detector which covers \pm 0.35 in pseudo-rapidity and $\pi / 2$ in azimuthal angle $\phi$ around the beam line. Events with collision points within $\pm 5 \mathrm{~cm}$ from the center of the detector along the beam line were analyzed. The collision centralities were classified by the correlation between BBC charge sum and ZDC energy sum[4]. We have checked the detector stability rigorously for a run range we have analyzed.

In addition, the dead or inefficient areas in the detector have been identified and the effects on the NBD parameters were carefully studied. We have estimated the relation between input $k$ values of NBD and biased $k$ values due to dead or inefficient areas for each size of pseudo-rapidity interval in each centrality. As long as the baseline distribution is known as NBD, one can generate the number of particles based on the probability distribution in all fine $\eta-\phi$ bins respectively. Dead maps can be produced from the track projection points in $\eta-\phi$ plane in the real data and the maps were used to suppress particles which hit the dead areas defined in the maps. After applying the dead maps, the biased multiplicity distributions were fit with NBD in given rapidity intervals again, which provides correction factors between the true $k$ values and the biased ones. Therefore systematic errors arise from the definition of the dead maps. As a general tendency, the dead maps scales only the absolute values of $k$, but not change the correlations between $k$ and rapidity interval sizes drastically. 


\section{Results}

Fig. 1 shows the charged particle multiplicity distributions in each pseudo-rapidity interval from $1 / 8$ to $8 / 8$ of the full rapidity coverage of $|\eta|<0.35$ with $0-10 \%$ events in the collision centrality. The distributions are shown as a function of the number of tracks $n$ normalized to the mean multiplicity $\langle n\rangle$. The vertical error bars show the statistical errors. The solid curves were determined by performing the NBD fit. In the following analysis, we have performed the NBD fit in each pseudo-rapidity interval size from $1 / 32$ to $32 / 32$ of the full rapidity coverage of 0.7 to determine a function shape in $k$ vs. $\delta \eta$ more precisely. The mean and RMS of reduced $\chi^{2}$ values in the NBD fit over all centralities and all interval sizes were obtained as 0.69 and 0.72 respectively, which corresponds to typically $95 \%$ confidence level. Therefore it is good enough to assume NBD as a baseline multiplicity distribution to obtain the integrated correlation function through the $k$ parameter based on Eq.(3.8).

Fig. 2 shows corrected $k$ parameters as a function of pseudo-rapidity interval sizes for centrality classes indicated inside the figure. The upper and lower two panels correspond to $10 \%$ and 5\% centrality bin width cases, respectively. The vertical error bars show the statistical errors and boxes show the systematic errors which come from correction factors on $k$ due to the possible variation of dead or inefficient areas in the tracking detector. The solid line indicates the fit result by using Eq.(3.8) with errors of quadratic sum of the statistical and systematic errors. The fit was performed from 0.02 to 0.7 in pseudo-rapidity. The lowest centrality bin was determined as 55-65\%. The fits are remarkably well resulting reduced $\chi^{2}$ of 0.44 at the worst which corresponds to above $99 \%$ confidence level. This guarantees that the parametrization is actually reasonable.

Fig.3 a), b) and c) show obtained fit parameters $\alpha, \beta$ and $\xi$ as a function of the number of participants $N_{p}$ where results for both $10 \%$ and $5 \%$ centrality bin width cases are plotted as red and blue circles respectively. $N_{p}$ was obtained from the centrality classes based on the Glauber model which is explained in [12] in detail. The horizontal errors correspond to ambiguities on the mean values of $N_{p}$ when the centralities are mapped upon $N_{p}$. The vertical error bars are obtained from errors on the fitting parameter by the Minuit program.

Fig. 4 shows the product of static susceptibility and corresponding temperature $\chi_{k=0} T$ as a function of the number of participants $N_{p}$ in the case of $10 \%$ centrality bin width. This quantity is proportional to ${\overline{\rho_{1}}}^{2} \alpha \xi$ based on Eq.(2.11), where ${\overline{\rho_{1}}}^{2}$ is normalized to 1.0 in the $0-10 \%$ centrality as defined in Eq.(3.9). The horizontal errors correspond to ambiguities on the mean values of $N_{p}$ when the centralities are mapped upon $N_{p}$. The errors on $\chi_{k=0} T$ were estimated by taking the error propagation between the three parameters into account.

\section{Discussions}

Since the $\alpha$ parameter corresponds to the correlation strength at the zero rapidity interval, the correlation is expected to be dominated by the Bose-Einstein effect in a short range as demonstrated by [11]. Since the observed chaoticity parameter $\lambda$ is almost constant as a function of $N_{p}$ in $\mathrm{Au}+\mathrm{Au}$ collisions at $\sqrt{S_{N N}}=200 \mathrm{GeV}$, the assumption of a constant $\alpha$ over all centrality samples might be more reasonable rather than leaving $\alpha$ as a free parameter within the accessible $\delta \eta$ region. This is because Eq.(3.8) indicates that $k$ can be approximated as $1 /(2 \alpha \xi / \delta \eta+\beta)$ in the limit of $\xi / \delta \eta<<$ 


\section{PHENIX Preliminary}
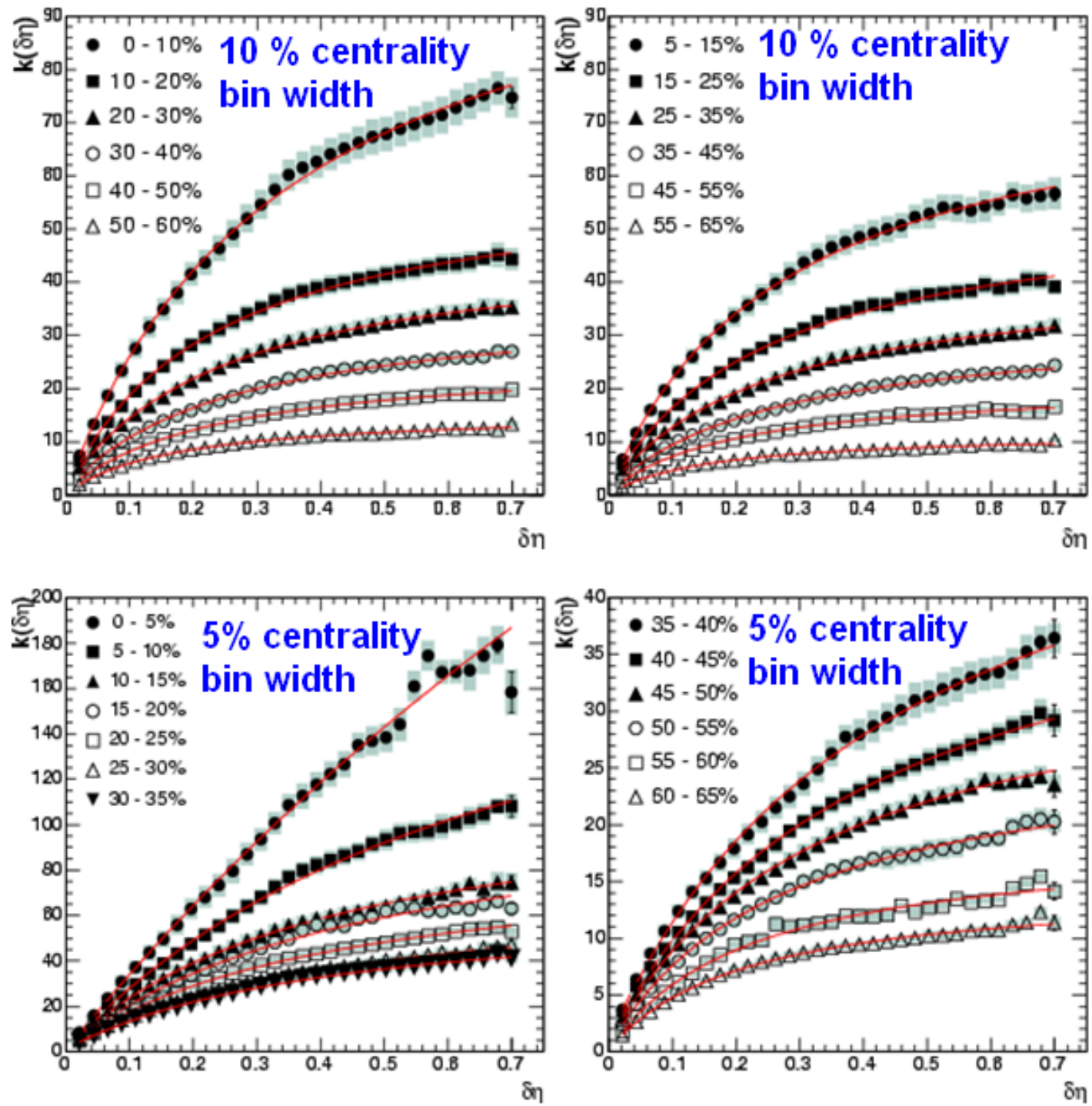

Figure 2: Corrected $k$ parameters as a function of pseudo-rapidity interval sizes for centrality classes indicated inside the figure. The upper and lower two panels correspond to $10 \%$ and $5 \%$ centrality bin width cases, respectively. The vertical error bars show the statistical errors and boxes show the systematic errors which come from correction factors on $k$ due to the possible variation of dead or inefficient areas in the tracking detector. The solid line indicates the fit result by using Eq.(3.8) with errors of quadratic sum of the statistical and systematic errors. The fit was performed from 0.02 to 0.7 in pseudo-rapidity. The lowest centrality bin was determined as $55-65 \%$. 

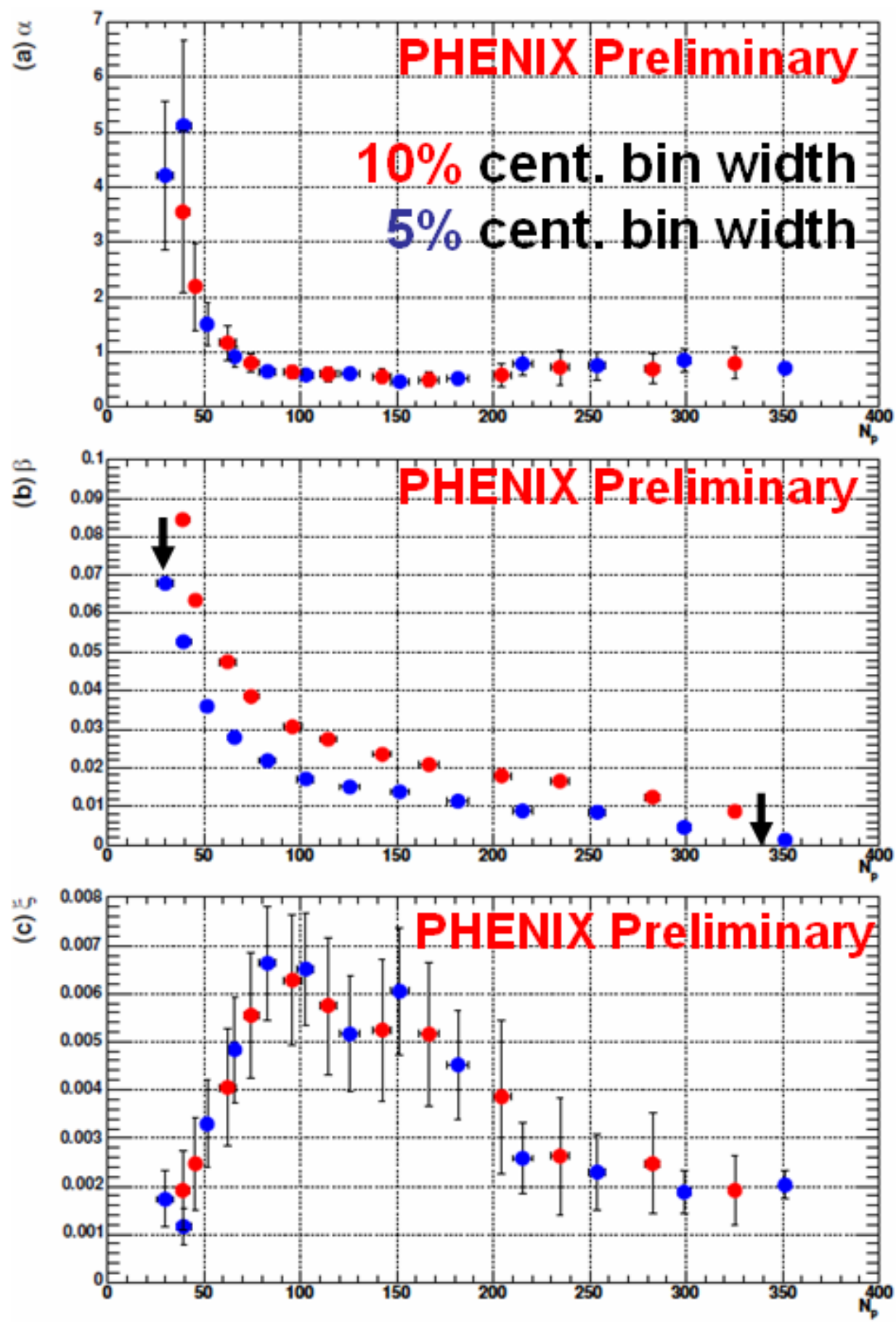

Figure 3: Extracted fit parameters $\alpha, \beta$ and $\xi$ as a function of the number of participants $N_{p}$ where results for both $10 \%$ and $5 \%$ centrality bin width cases are plotted as red and blue circles respectively. $N_{p}$ was obtained from the centrality classes based on the Glauber model which is explained in [12] in detail. The horizontal errors correspond to ambiguities on the mean values of $N_{p}$ when the centralities are mapped upon $N_{p}$. The vertical error bars are obtained from errors on the fitting parameter by the Minuit program. 


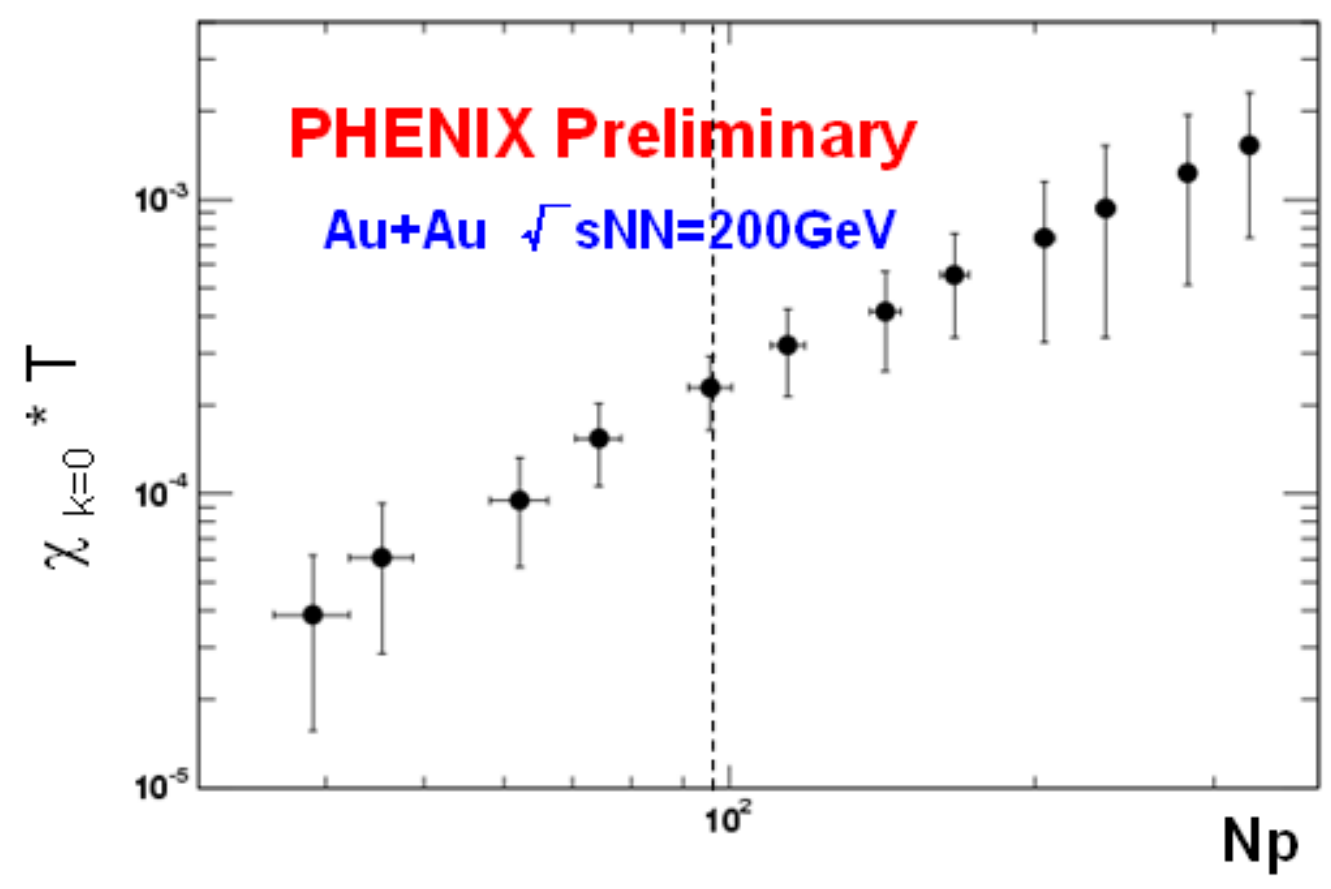

Figure 4: The product of static susceptibility and corresponding temperature $\chi_{k=0} T$ as a function of the number of participants $N_{p}$ in the case of $10 \%$ centrality bin width. This quantity is proportional to ${\overline{\rho_{1}}}^{2} \alpha \xi$ based on Eq.(2.11), where ${\overline{\rho_{1}}}^{2}$ is normalized to 1.0 in the $0-10 \%$ centrality as defined in Eq.(3.9). The horizontal errors correspond to ambiguities on the mean values of $N_{p}$ when the centralities are mapped upon $N_{p}$. The errors on $\chi_{k=0} T$ were estimated by taking the error propagation between the three parameters into account.

1 and there is an inevitable correlation between $\alpha$ and $\xi$ in the limit. Hence, unless the data points in very small $\delta \eta$ region are available, one can not completely neglect the correlation between $\alpha$ and $\xi$ in the fit with the integrated two particle correlation function. The direct measurement of two particle correlation function as demonstrated by [11] would resolve this issue in future.

It should be emphasized that the parametrization in Eq.(3.8) is robust and practically works, since the $\beta$ parameter can absorb the effect of centrality bin width effects. Fig. 3 b) shows the shift of $N_{p}$ dependence to lower values from $10 \%$ to $5 \%$ centrality bin width case, while other physically crucial parameters $\alpha$ and $\xi$ are rather stable. In other words, the $\beta$ parameter contains the effect from the fluctuations on $N_{p}$. The ambiguity on $N_{p}$ measured by PHENIX is not large compared to e.g. NA49 where only spectators from the projectile nucleus are measurable and it causes an increase of scaled variance of multiplicity distributions in peripheral collisions due to dominantly large $N_{p}$ fluctuations in the target nucleus. This is due to the partial sampling with respect to the total number of nucleons in two colliding nuclei. Since both projectile and target nuclei in both sides can be measured by BBC and ZDC at PHENIX, this kind of large ambiguities on $N_{p}$ is more suppressed even in peripheral collisions. Even if $N_{p}$ fluctuation is still remaining, $\beta$ parameter can absorb this kind of offset parts of fluctuations and the $N_{p}$ fluctuation is not harmful for the measurement of correlation lengths at all, since correlation lengths are based on the differential of fluctuations. In addition, $\beta$ would contain effects from the azimuthal correlation such as elliptic 
flows, since the PHENIX does not cover the full azimuth, fluctuations caused by reaction plane rotations and elliptic flows should be contained in principle. However, since the flow effect looks constant within the experimental accuracy in the pseudo-rapidity direction over unit rapidity[13], the measured correlation lengths in the pseudo-rapidity direction is not expected to be affected by elliptic flows or initial geometrical biases owing to the $\beta$ parameter.

Concerning the relation between $N_{p}$ and temperature, it is natural to assume that $N_{p}$ can be a monotonically and continuously increasing function of the initial temperature at least in the midrapidity region, since $d E_{T} / d \eta$ per participant pair is slowly raising as a function of $N_{p}$ as already observed in several RHIC energies[4]. The increase of the correlation length is seen at $N_{p} \sim 100$ and the corresponding energy density based on the Bjorken picture is $\varepsilon_{\mathcal{B}} \tau \sim 2.5 \mathrm{GeVfm}^{-2}$ which has been measured by PHENIX[4]. It is interesting to note that the energy density coincides with the one where the first drop of $J / \psi$ suppression from the normal nuclear absorption was observed at SPS[14], though the consistency of $E_{T}$ scales between two experiments must be carefully checked.

\section{Summary}

The multiplicity distributions measured in $\mathrm{Au}+\mathrm{Au}$ collisions at $\sqrt{s_{N N}}=200 \mathrm{GeV}$ are found to be well described by the negative binomial distribution. The two point correlation lengths have been extracted based on the function form by relating pseudo-rapidity density fluctuations and the Ginzburg-Landau theory up to the second order term in the free energy with the scalar order parameter. The function form can fit $k$ vs. $\delta \eta$ in all centralities remarkably well. The correlation lengths as a function of the number of participants $N_{p}$ indicate a non monotonic increase at around $N_{p}=100$. This could be a symptom of a critical behavior. Within the present systematic errors, the product of the static susceptibility $\chi_{k=0}$ and the corresponding temperature $T$ does not show an obvious turning point at the same $N_{p}$ where the correlation length increases.

\section{References}

[1] K. Adcox et al. [PHENIX Collaboration], Nucl. Phys. A 757, 184 (2005) [arXiv:nucl-ex/0410003].

[2] M. A. Stephanov, Prog. Theor. Phys. Suppl. 153, 139 (2004) [Int. J. Mod. Phys. A 20, 4387 (2005)] [arXiv:hep-ph/0402115].

[3] K. Adcox et al. [PHENIX Collaboration], Nucl. Instrum. Meth. A 499, 469 (2003).

[4] S. S. Adler et al. [PHENIX Collaboration], Phys. Rev. C 71, 034908 (2005) [Erratum-ibid. C 71, 049901 (2005)] [arXiv:nucl-ex/0409015].

[5] V. L. Ginzburg and L. D. Landau, Zh. Eksp. Teor. Fiz. 20, 1064 (1950).

[6] L. S. Ornstein and F. Zernike", Proc. Sect. Sci. K. med. Akad. Wet. 17, 793 (1914).

[7] D. J. Scalapino and R. L. Sugar, Phys. Rev. D 8, 2284 (1973).

[8] E. A. De Wolf, I. M. Dremin and W. Kittel, Phys. Rept. 270, 1 (1996) [arXiv:hep-ph/9508325].

[9] A. Bialas and R. Peschanski, Nucl. Phys. B 273, 703 (1986); A. Bialas and R. Peschanski, Nucl. Phys. B 308, 857 (1988).

[10] W. A. Zajc, Phys. Lett. B 175, 219 (1986). 
[11] M. J. Tannenbaum [E802 Collaboration], Nucl. Phys. Proc. Suppl. 71, 297 (1999).

[12] S. S. Adler et al. [PHENIX Collaboration], Phys. Rev. Lett. 91, 072301 (2003) [arXiv:nucl-ex/0304022].

[13] B. B. Back et al. [PHOBOS Collaboration], Phys. Rev. C 72, 051901 (2005) [arXiv:nucl-ex/0407012]; E. B. Johnson, arXiv:nucl-ex/0601010.

[14] B. Alessandro et al. [NA50 Collaboration], Eur. Phys. J. C 39, 335 (2005) [arXiv:hep-ex/0412036]. 